\title{
UNA APROXIMACIÓN EMPÍRICA A TRAVÉS DE LAS RELACIONES CIENCIA-TECNOLOGÍA-SOCIEDAD (CTS) EN ESTUDIANTES DE SECUNDARIA Y UNIVERSITARIOS VALENCIANOS
}

\author{
BORREGUERO, P. y RIVAS, F. \\ Departamento de Psicología Evolutiva y de la Educación. Facultad de Psicología. Av. Blasco Ibáñez, 21. \\ 46010 Valencia.
}

\section{SUMMARY}

The study reveals the Science-Technology-Society (STS) outlooks of high school students and university students. The VOSTS inventory (Aikenhead et al., 1989) was used to reflect The STS beliefs, positions and literacy. The outcomes of the study point to the need of introducing new concepts and attitudes, emphasizing epistemology, information and cognition.

\section{LA PROBLEMÁTXCA DE LA INTERACCIÓN CIENCIA-TECNOLOGIA-SOCIEDAD (CTS)}

Mientras que el debate sobre la educación científica gana una importancia substancial en todo el mundo, la perspectiva ciencia-tecnología-sociedad (Science-Technology-Society) se convierte en una de las aproximaciones más realistas con una influencia cada vez más presente en campos como la fillosofía, psicología, sociología y educación científica. El movimiento CTS nació a principios de los setenta, principalmente en los países de habla inglesa. La crisis económica hizo sonar alarmas sociales sobre algunos aspectos ecológicos como, por ejempio, los efectos colaterales de algunos bactericidas y la guerra del Vietnam, y fueron éstos algunos de los factores que propiciaron las primeras posturas antiestablishment y, en general, el resurgir de nuevas posiciones y actitudes ante el avance imparable de la socie. dad moderna. Como resultado de estas nuevas creencias, surgieron en algunas facultades y universidades anglosajonas nuevas disciplinas como, por ejemplo, la Histo. ria y Filosofía de la Tecnología, que intentaban analizar la compleja interacción que existe entre la ciencia, tecnología y la sociedad. Desde aquellas décadas, se ha realizado mucha investigación que abarca tanto el dise. no curricular como el análisis de las actitudes o implicaw ciones socio-políticas de estas posiciones, y una nueva forma de vivir la ciencia y el progreso se ha instaurado definitivamente en la escuela y en la opinión pública.

Mientras tanto, los propios gobiernos e industrias, envueltos en una economía de mercado con una competitiviđad en alza, necesitan técnicos bien formados y a la vez creativos, aunque se dé la paradoja de que la mayoría de los estudiantes se inclinan, cada vez más, por carreras de corte social o de letras, disminuyendo el interés por la preparación técnica y científica.

También la psicología de la educación se orienta dentro de este movimiento con todas sus implicaciones, inten tando reflejar tanto los cambios actitudinales necesarios para ja comprensión de la ciencia y la tecnoiogia como las posibles aplicaciones curriculares y educativas. Siguiendo el trabajo de Fleming (1989), el objetivo principal de la educación científica sería capacitar a los ciudadanos para participar en la torma de decisiones de los gobiernos democráticos, ejerciendo un rol activo en la creación de una sociedad mejor. Con el fin de capacitar a los ciudadanos para ejercer ese rol nos serviríamos de 
los siguientes medios: ampliación del conocimiento, cambiar las concepciones erróneas, y favorecer una mejor comprensión đe la naturaleza de la ciencia y la tecnología. Es decir, una formación científica adecuada supondría lograr una alfabetización científica y tecnológica, tanto en la profundización de los conocimientos como en el desarrollo de unas actitudes realistas, que capacite a los ciudadanos a desarrollar nuevos esquemas, nuevas pautas de comportamiento, en vez de reproducir viejos modelos sociales o supuestas verdades incuestionables.

Siguiendo la teoría de Ajzen y Fishbein (1980) de la acción razonada, la fuente principal de cambio social son las creencias y, cambiando estas creencias, podemos producir un cambio comportamental. Asumiendo esta teoria, para preparar a los estudiantes a tomar decisiones en el mundo de la sociotecnología (Fleming, 1989), necesitamos asesorar a los estudiantes en sus actitudes y creencias en los temas CTS. Por lo tanto, el primer paso, sería detectar cuáles son las actitudes y creencias de los estudiantes (Aikenhead et al., 1987 y Fleming, 1987) para establecer un punto de partida y reflejar la situación actual en nuestro país. Actualmente ya se han hecho varios estudios en Israel, Estados Unidos y Canadá (Aikenhead et al., 1987; Fleming, 1987, 1988, Zoller et al., 1991, Ben-Chaim et al., 1991 y Rubba et al., 1993); sin embargo, no tenemos conocimiento de ningún estudio experimental realizado en España que refleje fielmente las actitudes y creencias de los estudiantes ante los temas CTS, a excepción del trabajo de Solbes y Vilches (1992). Por lo tanto, el propósito fundamental de este estudio es reflejar la situación actual de los estudiantes universitarios en la Comunidad Valenciana, comparando los perfiles de los estudiantes de secundaria de la rama de ciencias con el de los estudiantes universitarios que estudian carreras científicas y tecnológicas (Arquitectura, Matemáticas e Ingeniería), subrayando la importancia de estos últimos como «futuros científicos y profesores» y su papel en una sociedad tecnológica.

\section{FINALIDAD DEL ESTUDIO}

El objetivo general que guía el estudio se concreta en las siguientes cuatro cuestiones:

1) Establecer la distribución de respuestas de los estudiantes bachilleres y universitarios en las posiciones, creencias y alfabetización CTS, distinguiendoentre aquelias perspectivas realistas y valiosas y aquellas erróneas y sin fundamento.

2) Comparar los niveles de alfabetización tecnológica y científica de ambos colectivos de estudiantes y su adecuación para la sociedad actual.

3) Analizar la influencia de los estudios universitarios en los estudiantes, si existe algún indicador en el sentido CTS.

4) Reinterpretar los principales factores teóricos de los ítems CTS que constituyen el estudio.

\section{METODOLOGÍA}

La muestra de la investigación consistió en 296 estudiantes de BUP ( $167 \mathrm{de} 2^{\circ}$, y 129 de $3^{\circ}$, y 172 estudiantes universitarios de $4^{8}$ y $5^{2}$ de carrera de las facultades de Arquitectura, Ingeniería y Matemáticas. En total 159 varones y 103 mujeres de las ciudades de Alicante, Castellón y Valencia.

Los instrumentos utilizados tradicionalmente, que emplean las escalas tipo Lickert, presentan un elevado grado de ambigüedad en la lectura y en la interpretación de los resulfados, asumiendo el investigador unas interpretaciones que muchas veces están lejos de las intenciones y atribuciones que realizan los sujetos. Para eliminar este tipo de ambigüedades, se desarrolló en Canadá, patrocinado por el Consejo de Educación Científica y Tecrológica, el VOSTS, formato 5 (Aikenhead G., Ryan A. y Fleming R, 1989), el primer instrumento construido empíricamente para medir actitudes y creencias en los temas CTS. Todas Ias alternativas de cada ítem que configuran el inventario han sido obtenidas a través de las respuestas de más 6.000 estudiantes, de tal modo que en cada ítem esta representado todo el abanico de posibles respuestas a esa cuestión. De este modo, la elección de una alternativa supone una posición clara y definida frente a un cuestión especifica, en la cual el sujeto identifica su respuesta entre el universo de respuestas que han dado los sujetos participantes a esa cuestión. Puesto que el cuestionario del VOSTS es una combina. ción de ítems que ha demostrado ser válida para este fin (Aikenhead, 1987 b, c, Aikenhead et al., 1987, Brunkhorst, 1987, Zoller et al., 1990 a), cualquier combinación de ítems de este cuestionario es válida y pueden ser utlizados los ítems independientemente unos de otros. En términos generales, y siguiendo las indicaciones de los autores, la validez de las cuestiones yace en la confianza que Ios investigadores han depositado en el proceso brevemente descrito (Aikenhead y Ryan, 1992).

\section{El VOSTS: adaptación}

El instrumento de investigación consistió en un cuestionario que comprende seis preguntas seleccionadas (de la uno a las seis) del inventario del VOSTS (Aikenhead, $1987 \mathrm{~b}$ ), y cuatro ítems más (Alfacien 2), elaborados por los autores siguiendo la metodología e indicaciones para los ́́tems desarrollados empíricamente (Aikenhead et al., 1992). Los ítems completos del VOSTS y de Alfacien 2 han sido resumidos en este artículo y el estudio completo puede verse en Borreguero (1992).

Los ítems del VOSTS y los propios (Alfacien 2) se seleccionaron de acuerdo con nuestros criterios, en concordancia con estudios anteriores (Ben-Chaim et al., 1991, Zoller et al., 1991) y siguiendo esta clasificación:

1) Posiciones conceptuales CTS: Esta categoría se relaciona con el conocimiento conceptual, enfatizando la cognición sobre la actiłud (Zoller et al., 1991). Ítems 1, 2 (VOSTS 1.7 y 4.1 ) y 8 (Alfacien 2 ). 
2) Actitudes/creencias. Son predictores de la conducta futura en los temas CTS (Zoiler et al., 1991). Items 3 y 6 (VOSTS 11.2, 20.1). Ítem 9 (Alfacien 2).

3) Alfabetización científica. Esta categoría incluye: a) comprender las interacciones CTS; $b$ ) asesoramiento tecnológico; y c) capacidad de participar en las democracias tecnodependientes (Zoller et al., 1991). Ítems 4 y 5 (VOSTS 12.1 y 18.1). Ítem 7,10 (Alfacien 2).

Los datos presentados constituyen Ia base de la distribución de las respuestas de los estudiantes universitarios contrastados con los estudiantes de secundaria (segundo y tercero de BUP). Se realizó una prueba chi-cuadrado $(p=0,05)$ entre los perfíles de los grupos comparados en los diez ítems de los cuestionarios utilizando los porcentajes totales de las respuestas. Los porcentajes totales incluyen los porcentajes de las tres últimas alternativas de cada ítem (no entiendo la problemática, no se bastante de este tema, ninguna se adecua a mi punto de vista...), denominadas respuestas "pasivas»; el resto de las alternativas que reflejan una postura clara y definida se considerarían las respuestas «activas».

En términos generales, los porcentajes de respuestas "pasivas» están todos por debajo del veinte por ciento, excepto en el caso del ítem 8 . La prueba chi-cuadrado desciende a cada alternativa de los diez ítems del inventario, de tal modo que los resultados nos indican la distribución de las respuestas de Ios grupos experimentales. Los resultados se interpretaron en términos de diferencias intersignificativas e intrasignificativas o diferencias no significativas entre estudiantes universitarios y bachilleres. Para obtener la distribución normalizada de las variables, las frecuencias acumulativas se transformaron en puntuaciones $z$. Este procedimiento especial permitió realizar una prueba $\mathrm{F}$ y un análisis factorial de Ias variables. Los resúmenes siguientes reflejan los diez ítems del estudio, con las alternativas a elegir. A los estudiantes se les pedía que eligiesen una alternativa de cada ítem. Las tres últimas alternativas son las mismas para cada átem.

\section{RESULTADOS Y COMENTARIOS}

Los resultados de los perfiles CTS de universitarios y bachilleres en la prueba chi-cuadrado se muestran en las tablas I a X.

\section{TABLAS DE DISTRIBUCIÓN CHI-CUADRADO Y ANÁLISIS DE VARIANZA}

Tabla I

Resumen de respuestas ítem 1 . Posiciones CTS.

Los científicos e ingenieros deberian decidir en la distribucion mundial de los alimentos.

( $\mathrm{B}=$ Bachillerato, $\mathrm{U}=$ Universidad).

Cicntíficos e ingenieros deben decidir porque:

$\begin{array}{cc}\text { Chi-cradrado } & (p=0,05) \\ \text { B } & \text { U } \\ & \\ 8 & 1 \\ 11 & 11 \\ 16 & 9 \\ 48^{*} & 66^{*} \\ 2^{*} & 5^{*} \\ 3 & 1 \\ 0 & 0 \\ 6 & 4\end{array}$

Tabla II

Resumen de respuestas ítem 2. Posiciones CTS.

Los cientificos españoles deben ser responsables del daño que produzcan sus descubrimientos porque:
A. Es parte del trabajo científico.
B. Deben hacer buen uso y abandonar el mal uso.
C. Deben ser conscientes de los efectos posibles.
D. La responsabilidad debe ser compartida.
E. Las personas que los usar son responsables.
F. Los resultados no se pueden predecir.
G. De este modo, los científicos no investigarían.
H. La ciencia y la moral son cosas distintas.
Otras tespuestas (I,J.K.).

\begin{tabular}{cc}
\multicolumn{2}{c}{ Chi-cuadrado } \\
B
\end{tabular}


Tabja III

Resumen de respuestas ítem 8. Posiciones CTS.

En España, debería aumentarse el número de horas dedicadas a ciencia y tecnología en educación primaria y secundaria.

$\begin{array}{cc}\text { Chi-cuadrado } \\ \text { B } & \%=0,05) \\ & \text { U } \\ 7 & 5 \\ 22 & 22^{*} \\ 22 & 26 \\ 9 & 4^{*} \\ 3 & 2 \\ 6 & 4 \\ 5 & 3 \\ 18^{*} & 31^{*}\end{array}$

Tabla IV

Resumen de respuestas f́tem 3. Posiciones CTS.
B. Se debería mejorar la educación científica de los estudiantes.
tion ciéfica adecuada.
F. Deberíamos seguir con nuestra tradición humanística.
G. El país no está interesado en la educación.
Ottas respuestas (H.I.J.).

3. La tecnología es principalmente:
A. Parecido a la ciencia.
B. La aplicación de la ciencia.
C. Nuevos procesos, instrumentos.
D. Bombas, aparatos militares, etc.
E. Robótica, electrónica, ordenadores, etc.
F. Una técnica para bacer cosas.
G. Un modo de resolver problemas prácticos.
H. Inventar, diseñar cosas...
Otras respuestas (1.J.K.).

\begin{tabular}{cc}
\multicolumn{2}{c}{ Chi-cuadrado } \\
B & $\%=0,05)$ \\
& $\mathrm{U}$ \\
3 & \\
3 & 0 \\
43 & 3 \\
$23^{*}$ & 42 \\
3 & $34^{*}$ \\
8 & 2 \\
3 & 8 \\
1 & 5 \\
5 & 1 \\
& 5
\end{tabular}

Tabla V

Resumen de respuestas ftem 6. Creencias CTS.

6. Los cientificos proyectan estas características (honestidad, tolerancia, etc.) más que los demás en su trabajo.
A. Proyectan más estas características para que no ocurran desastres.
B. Las proyectan más por la naturaleza de su trabajo.
C. Todo el mundo debe tener estas características.
D. Proyectan estas características menos.
E. Porque han sido influidos por compañias o gobiernos.
Otras respuestas (F.G.H.).

Tabla VI

Resumen de respuestas ftem 9. Creencias CTS.

9. La investigación cientifica y tecnológica debe ser totalmente libre.

A. Los cientifficos no pararán de investigar sin importar las consecuencias.

B. No son responsables de los resultados de la investigación.

C. Seria imposible establecer reglas regulando las normas.

D. Es necesario informar a los ciudadanos de los riesgos potenciales.

E. Los ciudadanos deben controlas sólo st posible aplicación.

F. Hoy en día, apenas se puede confiar en sus beueficios.

G. Es sospechoso cómo usan esta información tos políticos.

Otras respuestas (H.I.J.).

$\begin{array}{cc}\text { Chi-cuadrado } & (p=0,05) \\ \text { B } & \text { U } \\ & \\ 14 & 8^{*} \\ 7 & 14 \\ 4 & 1 \\ 42^{*} & 58^{*} \\ 5 & 7 \\ 11 & 5\end{array}$


Tabia VII

Resumen de respuestas ítem 4. Alfabetización CTS

Seria mejor invertir en investigación tecnológica que en cientifica.

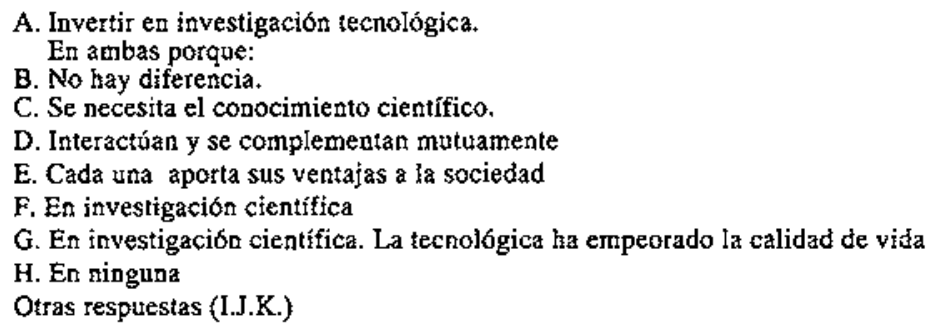

$\begin{array}{cc}\underset{\mathrm{B}}{2} \mathrm{Chi}-\mathrm{cuadrado} \\ & (\mathrm{p}=0,0 \mathrm{U}) \\ 4 & 6 \\ 28 & 36 \\ 17^{*} & 26^{*} \\ 24 & 21 \\ 2 & 4 \\ 2 & 0 \\ 2 & 1 \\ 2 & 1 \\ 6 & 3\end{array}$

Tabia VIII

Resumen de respuestas f́tem 5. Alfabetización CTS.

Cuando los cientificos no están de acuerdo es porque:
A. Porque no se han descubierto todos los hechos.
B. Porque los científicos conocen hechos diferentes.
C. Debido a teorías cientifficas diferentes.
D. Debido a diferentes valores y opiniones.
E. Por diferentes razones: falta de información, teorias diferentes...
F. Sobre todo por opiniones personales, valores morales, etc.
G. Porque han sido influidos por compañías o gobiernos.
Otras respuestas (H.I.J.).

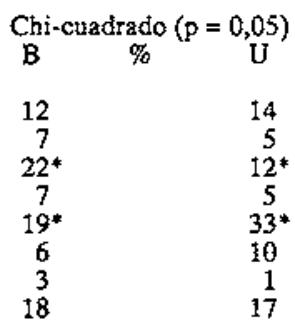

Tabla IX

Resumen de respuestas item 7 . Alfabetización CTS

La industria española parece desconfiar de su propia investigacián:

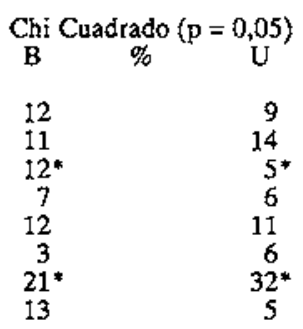

Tabla X

A. Se debe a la falta de inversión en la investigación.

B. Porque algunos países están más desarrollados.

C. Hay una apatía general hacia la investigación cientifica.

D. Las fundaciones y universidades españolas son desconocidas como productoras de investigación.

E. El país que no invierte en investigación dependerá de otros.

F. España pronto realizará contribuciones importantes.

G. El gobierno debería diseñar programas rentables de investigación.

Otras respuestas (H.I.J.)

ítem 10. Alfabetización CTS.

10. Los medios de comunicación frecuentemente anuncian descubrimientos científicos que constantemente crean nuevas controversias. El ciudadano comun:

$\begin{array}{cc}\text { Chi-cuadrado } & (p=0,05) \\ \text { B } & \% \\ & \text { U } \\ 15 & 11 \\ 26 & 24 \\ 14^{*} & 34^{*} \\ 4 & 2 \\ 5 & 3 \\ 4 & 6 \\ 4 & 4 \\ 18 & 13\end{array}$


Tabla XI

Pruebas F entre $2^{\circ}$ de BUP, $3^{*}$ de BUP y universitarios.

\begin{tabular}{ccccccccc} 
Pregunta & F & P & Pregunta & F & P & Pregunta & F & P \\
\hline 1 & 0,99 & 0,62 & 2 & 7,21 & $0,00^{*}$ & 3 & 0,99 & 0,62 \\
4 & 4,57 & $0,01^{*}$ & 5 & 0,95 & 0,061 & 6 & 3,42 & $0,03^{*}$ \\
7 & 2,50 & 0,08 & 8 & 1,00 & 0,63 & 9 & 0,66 & 0,52 \\
10 & 0,05 & 0,95 & & & & & & \\
\hline
\end{tabular}

Tabla XII

Componentes principales.

\begin{tabular}{ccc} 
Factor & Eigenvalue & $\%$ de la varian \\
\hline 1 & 2,12 & 21,2 \\
2 & 1,13 & 32,5 \\
3 & 1,05 & 43,0
\end{tabular}

$\begin{array}{ccccc}\text { Saturaciones } & & & & \\ & \text { Factor I } & \text { Factor II } & \text { Factor III } & \text { h } \\ 1 & 0,416 & -0,172 & 0,454 & 4,1 \\ 2 & -0,142 & 0,055 & 0,828 & 7,1 \\ 3 & -0,171 & 0,732 & 0,045 & 5,7 \\ 4 & 0,294 & 0,590 & -0,095 & 4,4 \\ 5 & 0,207 & 0,573 & 0,184 & 4,1 \\ 7 & 0,583 & 0,011 & 0,048 & 3,4 \\ 8 & 0,507 & 0,050 & 0,165 & 2,9 \\ 9 & 0,554 & 0,283 & 0,122 & 4,0 \\ 10 & 0,633 & 0,107 & -0,089 & 4,2 \\ & & & & 12\end{array}$

Son importantes las diferencias significativas halladas en las posiciones CTS en las preguntas 1 y 2. Por un lado, la mayoría de estudiantes universitarios seleccionan una posición participativa (D, 66\%, ítem 1); por el otro, rechazan cualquier responsabilidad en los descubrimientos (E, 33\%, f́tem 2). En el ítem 8, la distribución es bastante similar en ambos grupos, con un alto porcentaje de respuestas «pasivas» en los estudiantes universitarios $(31 \%)$, pero con un nivel aceptable para estudiantes de secundaria (18\%). Este hallazgo indica la necesidad de revisar la validez de esta cuestión.

En las cuestiones relacionadas con creencias CTS se encontraron diferencias significativas en el ítem 6 (Los científicos proyectan la honestidad, etc. en su trabajo...). En términos generales, la mayoría de los estudiantes piensan que los científicos proyectan estas características menos que otros trabajadores. Este porcentaje es más alto en universitarios que en bachilleres (U= $58 \%$ ), $\mathrm{B}=42 \%$ ). Al observar la distribución de estas creencias, bay un porcentaje muy bajo de estudiantes que consideran la tecnología como una aplicaciốn de la ciencia (ítem 3) en ambos grupos; en general, la mayoría de los estudiantes seleccionan alternativas que expresan puntos de vista fundamentados sobre las interacciones CTS, aunque no se encontraron diferencias significativas. En el ítem 9, la mayoría de los estudiantes en ambos grupos $(37 \%=\mathrm{B}, \mathrm{y} 40 \%=\mathrm{U})$ cree que los ciudadanos deberían ser informados sobre estos temas y no se hallaron diferencias significativas entre ellos.

Al analizar el nivel de alfabetización CTS, se encontraron diferencias significativas en algunas cuestiones, con porcentajes más altos de respuestas en la dirección adecuada en los estudiantes universitarios. El perfil de respuestas en el ítem 4 muestra que no existe diferencia- 
cion entre invertir en ciencia y tecnología en los dos grupos $(\mathrm{B}, \mathrm{E}=28 \%, \mathrm{U}, \mathrm{E}=36 \%$ ), aunque cada grupo tiene un perfil diferente de respuesta. Del mismo modo, en el ítem 5, los estudiantes universitarios consideran que los científicos interpretan los hechos de modos diferentes por una combinación de motivos distintos $(E=22 \%)$, mientras que los estudiantes de secundaria piensan que estas interpretaciones se deben a teorías diferentes $(E=22 \%)$. En la cuestión 7, la mayoría de los estudiantes culpa al gobierno de la situación actual, con un porcentaje más alto de universitarios en esta postura $(B=21 \%$, $U=32 \%$ ). Ambos grupos piensan mayoritariamente que los ciudadanos deberían ser informados $(\mathrm{H}, 37 \%, \mathrm{U}$, $40 \%$ ) y no se encontraron diferencias entre eilos.

La prueba $F$ confirma esta interpretación para los ítems $2,4,6$ y $7(p=0$. 08). La exposición de estudiantes universitarios a temas científicos se refleja claramente en sus perfiles CTS, aunque a veces en un direccion inesperada. Los ítems $3,8,9$ y 10 tienen una distribución muy similar y no se encontraron diferencias significativas en estas cuestiones. Este hallazgo indica la falta de una preparación adecuada en los temas CTS en aquellas carreras universitarias de corte científico y tecnológico.

Como se sugería anteriormente, los estudios universitarios favorecen la educación CTS en sólo algunos aspectos, sobre todo aquéllos relacionados con la participación democrática, la implantación de la ciencia y la interpretación de los hechos científicos. A pesar de que los estudiantes universitarios mantienen actitudes más realistas en estos aspectos, a veces su estatus de «futuros científicos» favorece actitudes en la dirección contraria, como en el ítem 2.

El último paso del análisis, el análisis factorial de componentes principales, reveló principalmente tres factores de la matriz de correlaciones, que explican el $43 \%$ de la varianza total:

a) Factor I: Aquellos ítems relacionados con actitudes de confianza/desconfianza en la investigación científica (explica el $17 \%$ de la varianza)

10. «Los medios de comunicación frecuentemente anuncian nuevos descubrimientos que cambian nuestra vida diaria.»

7. «La industria española no confía en su propia investigación.»

8. «Los cursos de ciencia obligatorios se deberían aumentar.»

9. «La investigación científica debería ser lo más libre posible.»

b) Factor II: Percepción de la ciencia y la tecnología (explica el 14\% de la varianza)

3. "La tecnología es principalmente...»

4. «Sería mejor invertir en investigación tecnológica...»
6. «Los científicos proyectan ciertas características personales...»

\section{c) Factor HI: Responsabilidad (explica el 12\% de la varianza)}

\section{1. «Los científicos e ingenieros deberían decidir.»}

2. «Los científicos deberían ser responsables del daño...»

3. «Las opiniones científicas no tienen nada que ver con los motivos personales."

Este análisis provee una nueva clasificación de los ítems basada en sus correlaciones, así como una nueva descripción psicológica de las variables.

\section{CONCLUSIONES}

La aplicación y desarrollo del VOSTS ha sido útil como punto de partida para: a) reflejar la situación actual en Ios temas CTS de la Comunidad Valenciana en bachilleres y universitarios; b) asesorar a profesores tanto universitarios como de secundaria en las orientaciones CTS de la enseñanza; y c) generar nuevos ítems CTS (Aikenhead et al., 1992), en este caso los ítems 7, 8, 9 y 10 (Alfacien 2). Los perfiles CTS de bachilleres y universitarios en la rama de ciencias puede servir como una primera aproximacion y marco de referencia para reflejar la situación actuaf y hacer comparaciones transculturales. El análisis de ambos perfiles suministra datos muy interesantes sobre la caracterización de estos perfiles a través del proceso de enseñanza y aprendizaje de la ciencia.

Los resultados del estudio sugieren que: a) hay un cambio en las perspectivas CTS como consecuencia de una mayor exposición de los estudiantes al proceso de ensenanza-aprendizaje de la ciencia; b) se encontraron diferencias significativas en las varianzas de los perfiles CTS en las tres categorías: posiciones CTS (ítem 2), creencias CTS (ítem 6), alfabetización CTS (ítem 4 y casi en el ítem 7); c) el cambio en las perspectivas CTS ocurre a veces a través de la «adoctrinación» en vez de la educación, como por ejemplo en el ítem 2 ; y d) hay diferencias menores en los perfiles de los otros ítems (3, $5,8,9$, y 10 ).

EI análisis de componentes principales sugiere la reinterpretación de los items CTS. Sobre la base de los resultados empíricos de las respuestas de los estudiantes podemos enumerar la existencia de tres factores que explican el $47 \%$ de la varianza: a) factor I: confianza / desconfíanza; b) percepción global de la ciencia y la tecrología; y c) responsabilidad. Los resultados del análisis indican tres nuevas dimensiones psicologicas que podrían ser tomadas en cuenta para estudios posteriores.

Los resultados del estudio indican la necesidad de establecer una formación CTS tanto en secundaria como en 
la universidad. La ausencia de unas creencias y actitudes realistas en este campo predicen un pobre comporta- miento en el futuro. El proceso de enseñanza y aprendizaje de la ciencia, si no tiene en cuenta las metas CTS, no es suficiente para obtener unas posiciones fundamentadas y valiosas. Aunque el nivel de alfabetización CTS mejora con el nivel de estudios, en términos generales, todavía está lejos del nivel deseado. Si las concepciones erróneas $o$, más concretamente, la epistemología científica personal que revela el estudio son representativas, nos podemos cuestionar la adecuación de estas concepciones tanto en universitarios como en estudiantes de secundaria. Los estudiantes universitarios, futuros científicos o profesores universitarios, que trabajarán en los campos de arquitectura e ingeniería, todavía mantienen unos conceptos erróneos sobre las interacciones que existen entre la ciencia, tecnología y la sociedad. Como

\section{REFERENCIAS BIBLIOGRÁFICAS}

AIKENHEAD, G.S. (1987). High School graduates beliefs about Science-Technology. Society III: Caracteristics and limitations of scientific knowledge. Science Education, 71(4), pp. 459-487.

AIKENHEAD, G.S. FLEMING, R.W. y RYAN, A. (1987). High school graduates 'beliefs about science, technology and society I. Methods and issues in monitoring students views. Science Education, 71(2), pp. 145-161.

AIKENHEAD, G. y RYAN, A. (1992). The development of a new instrument: Views on Science-Technology-Society (VOSTS). Science Education, 76(5), pp. 477-491.

AJZEN, I. y FLAHBEIN, M. (1980). Understanding attitudes and predicting social behavior. Englewood Cliffs. NI: Prentice Hall.

BEN-CHAIM, D. y ZOLLER, U. (1991). The STS outlook profiles of Israeli high-school students and their teachers. International Journal of Science Education, 13(4), pp. 447458.

BORREGO, P. (1992). STS: Una aproximación empirica a las relaciones ciencia-tecnología-sociedad. Tesis de licenciatura. Departamento de Psicología Evolutiva y de la Educación. Valencia: Facultad de Psicología.

BARUNKHORAT, H.K. (1987). A comparison of student/ teacher position on selected science/technology/society topics: a preliminary study, en Requarts, K. (ed.), Science and technology education and the quality of life. Proceeding of the 4 th International Symposium on World trends.

FLEMING, R.W. (1987). High school graduates 'beliefs about science, tecnology and society Il. The interaction among indica el estudio, el objetivo principal no es sólo dar a conocer a los estudiantes más ciencia, sino también introducir nuevos contenidos y orientaciones CTS, enfatizando la epistemología, la búsqueda de información y la cognición y -siguiendo a Kuhn (1993)-, acercando las estrategias del pensamiento científico a nuevas situaciones de carácter social que envuelven la vida diaria, aprovechando esa conducta exploratoria innata que poseen los niños y manteniéndola y avivándola durante la adolescencia. Todos los hallazgos de este estudio nos indican la necesidad de introducir nuevos temas sobre la interacción ciencia-tecnología-sociedad en los estudios secundarios y universitarios, desarrollando nuevos objetivos curriculares, enfatizando los aspectos actitudinales y diseñando cursos de reciclaje tanto para los profesores de secundaria como para los universitarios.

science-technology-society. Science Education, 71(2), pp. 163-186.

FLEMING, R.W. (1988). Undergraduate science students' views on the relationship between science, technology and society. International Journal of Science Education, 10(4), pp. $499-463$.

FLEMING, R.W. (1989). Literacy for technological age. Science Education, 73(4), pp. 391-404.

HOLMAN (1989). Science-technology-society education. International Journal of Science Education, 10(4), pp. 343-345.

RUBBA y HARKNESS, W.L. (1993). Examination of preservice and in-service secondary science teachers ${ }^{*}$ beliefs about science-technology-society interactions. Science Education, 77(4), pp.407-431 (1993).

SOLBES, J. y VILCHES, A. (1992). EI modelo constructivista y las relaciones ciencia-técnica y sociedad. Enseñanza de las Ciencias, 10(2), pp. 181-186.

ZOLLER, V., EBEEZER, J., MORELY, K, PARAS, S., SANDBERG, V., WEST, C., WOLTHERE, T. y TAN, S.H. (1990). Goal attainment in Science-Technology-Society Education and reality: The case of British Columbia. Science Education, 74(1), pp. 19-36.

ZOLLER, U., DONN, S., WILD, R., BECHKETT, P., (1991). Students versus their teachers' beliefs and positions on science-technology-society oriented issues. Intermational Journal of Science Education, 13(1), pp. 25-36. 